\title{
EFFICACY OF HERBICIDES IN POTATO CROP
}

\author{
Alicja Baranowska', Iwona Mystkowska', Krystyna Zarzecka², Marek Gugała² \\ 1 Institute of Agriculture, Pope John II State School of Higher Education in Biala Podlaska, Sidorska 95/98, \\ 21-500 Biała Podlaska, Poland, e-mail: alabar@tlen.pl \\ 2 Department of Agrotechnology, University of Natural Sciences and Humanities in Siedlce, B. Prusa 14, 08- \\ 110 Siedlce, Poland
}

Received: 2015.11.20

Accepted: 2015.12.09

Published: 2016.01.06

\begin{abstract}
The experiment was conducted on slightly acidic soil classified as very good rye complex. The aim of the study was to assess the impact of plant-care procedures with the use of herbicides and their mixtures on the number and weed species composition in the field of three edible potato cultivars: Satina, Tajfun and Cekin. The weed infestation was determined at two dates: before the row closure and before tuber harvest. The most effective in decrease of weed infestation, before the row closure as well as before the harvesting of the tubers, appeared to be variations in which herbicides mixtures were used: Command $480 \mathrm{EC} 0.2 \mathrm{l} \cdot \mathrm{ha}^{-1}+$ Dispersive Afalon $450 \mathrm{SC} 1.0 \mathrm{l} \cdot \mathrm{ha}^{-1}$ and Stomp $400 \mathrm{SC} 3.5 \mathrm{l} \cdot \mathrm{ha}^{-1}+$ Dispersive Afalon $450 \mathrm{SC} 1.0 \mathrm{l} \cdot \mathrm{ha}^{-1}$. According to the conducted researches, the potato cultivars did not have significant effect on weed infestation marked at the beginning and at the end of vegetation.
\end{abstract}

Keywords: herbicides, potato, weed control methods.

\section{INTRODUCTION}

The number of weed species occurring in arable crops in Poland ranges from 300 to 400 [Sobótka 1999], and on potato plantations shapes from 29 to 55 [Nowacki and Podolska 2005]. According to Rola [2002], $50 \%$ of potato cropping in Poland are weedy to a medium and large extent and the reappearance of weeds plays an important role. Among all pests, weeds are characterized by the highest potential ability to lowering yields, on average from 10 to $50 \%$ [Praczyk and Skrzypczak 2011]. However, according to Wesołowski and Kacuga [1989], lowering yields caused by weed infestation may run into $70 \%$, because of pests $-18 \%$, and diseases - on average $6 \%$.

The weed infestation of potato plantation enforces the need of looking for different weed control methods [Pytlarz-Kozicka 2002]. Using herbicides and their mixtures ensures high effectiveness of weed control which contributes to potato tuber yield increase [Guttieri and Eberlein 1997; Hashim et al. 2003]. Herbicides form a permanent element of the cultivation of crops technology. They guar- antee almost complete removing of most of the weed species which occur in the potato canopy. What is more, they also reduce the development of many diseases by the destruction of the source of infection [Giebel et al. 1992]. The application of chemical methods of maintenance, in comparison with mechanical ones, may reduce weed infestation up to 99\% [Zarzecka and Gugała 2004].

According to Pruszyński [2000], in the next ten, twenty years, the basis for protection of plants and reduction of losses caused by pests, including weeds, will be use of chemical plant protection products. Therefore, an attempt was made in this study to specify the impact of weed control methods as well as use of herbicides and their mixtures on the number and species composition in the canopy of three edible potato cultivars.

\section{MATERIAL AND METHODS}

The field researches were conducted in the years 2008-2010, at the Zawady Agricultural Experimental Station belonging to the University of 
Natural Sciences and Humanities in Siedlce. The experiment was set up according to the split-splot design in three replications. The experimental factors were as follows:

1) I factor - five weed control methods:

- mechanical weed control - control object, until emergence - earthing up 3-4 times and earthing-up with harrowing (earthing-up once, once or twice earthing-up connected with harrowing + earthing-up once), and earthing-up twice after emergence,

- mechanical and chemical control, until emergence earthing-up and two or three times with harrowing and Command $480 \mathrm{EC}$ about 7 days before emergence, at a dose of $0.21 \cdot \mathrm{ha}^{-1}$,

- mechanical and chemical control, earthing-up with harrowing until emergence and spraying with a mixture of herbicides Command 480 EC $0.21 \cdot \mathrm{ha}^{-1}+$ Dispersive Afalon 450 SC 1.0 $1 \cdot$ ha $^{-1}$ about 7 days before emergence,

- mechanical and chemical control, earthing-up with harrowing until emergence and herbicide Stomp 400 SC $3.51 \cdot$ ha $^{-1}$ about 7 days before emergence,

- mechanical and chemical control, earthing-up with harrowing before emergence and spraying with a mixture of herbicides Stomp 400 SC $3.51 \cdot \mathrm{ha}^{-1}+$ Dispersive Afalon 450 SC 1.0 $1 \cdot$ ha $^{-1}$ about 7 days before emergence,

2) II factor - three potato cultivars: Satina, Tajfun, Cekin.

A field experiment was conducted on soil classified in the division - autogenic soils, order - brown soils, type - grey-brown podzolic soils formed from light loamy sands and strong loamy sands, soil quality class IVa and IVb classified as very good rye complex, slightly acid. This soil was characterized by a high concentration in available phosphorous, high concentration in potassium and average concentration in magnesium.

Potato was cultivated in the field after winter cereals. Each year in autumn proceeding seeding the tubers, fertilizing with natural at a dose of $25 \mathrm{t} \cdot \mathrm{ha}^{-1}$ of stable manure as well as mineral phosphorus and potassium fertilization in the amount $\mathrm{P}=44.0 \mathrm{~kg} \cdot \mathrm{ha}^{-1}\left(\mathrm{P}_{2} \mathrm{O}_{5}=100 \mathrm{~kg}\right)$ i $\mathrm{K}=$ $124.5 \mathrm{~kg} \cdot \mathrm{ha}^{-1}\left(\mathrm{~K}_{2} \mathrm{O}=150 \mathrm{~kg}\right)$ were applied.

Each year in spring nitrogen fertilizers were applied at a dose of $\mathrm{N} 100 \mathrm{~kg} \cdot \mathrm{ha}^{-1}$. The potatoes were planted in the second and third ten-day period of April in row spacings $62.5 \times 40 \mathrm{~cm}$. In the experiment medium early varieties of edible pota- to were cultivated: Satin, Tajfun and Cekin. Plant protection treatments against diseases and pests were applied in accordance with the plant protection recommendations.

In order to compare efficiency of the weed control methods, species composition and the number of weeds per $1 \mathrm{~m}^{2}$ were determined. The measurements were conducted on three randomly chosen plot areas marked out by a frame $33.4 \times 150$ $\mathrm{cm}\left(5010 \mathrm{~cm}^{2}\right)$.

The analysis of the field weed infestation was conducted at two dates: 2-3 weeks after application of herbicides (before row closure of the crop) and at the end of potato vegetation (1-2 weeks before tuber harvest). Names of weeds were given in accordance with Atlas of Weed Plants published by The Plant Protection Institute in Poznań [Praczyk 2015].

The results of the study were statistically analysed using analysis of variance. The significance of variability sources was tested by means of Fisher-Snedecor's test, and evaluation of the significance of differences at a significance level of $\mathrm{p}=0.05$ between comparable means was performed using Tukey's multiple range test.

The weather conditions in the study years were changeable. According to hydrothermal Sielianinow's coefficient, analysed vegetation seasons were characterized by the absence of drought. The 2008 year was marked by favourable weather conditions, the rain was evenly distributed and temperatures were close to the average of multi-annual period. In 2009 the weather conditions in particular months were differential, ranged from strong drought in April and July, when Sielianinow's coefficient came to accordingly 0.26 and 0.44 , to the absence of drought in June (3.08). However, in the 2010 growing season, strong drought was observed when hydrothermal Sielianinow's coefficient was 0.40 and temperatures were close to the long-term means (Table 1).

\section{RESULTS AND DISCUSSION}

The weed infestation of potato plantation marked at two dates, in the initial period of vegetation and before tuber harvest, made it possible to compare all weed control methods and pointing at the best option of reducing the fresh weight of weeds. The number of weeds marked at the initial period of potato vegetation and before tuber 
Table 1. Rainfalls and air temperatures in 2008-2010 vegetation seasons at the Zawady Meteorological Station

\begin{tabular}{|c|c|c|c|c|c|c|c|}
\hline \multirow{2}{*}{ Years } & \multicolumn{6}{|c|}{ Months } & \multirow{2}{*}{$\begin{array}{l}\text { Mean/Sum } \\
\text { IV-IX }\end{array}$} \\
\hline & IV & $\mathrm{V}$ & VI & VII & VIII & IX & \\
\hline \multicolumn{8}{|c|}{ Rainfalls (mm) } \\
\hline 2008 & 28.2 & 85.6 & 49.0 & 69.8 & 75.4 & 63.4 & 371.4 \\
\hline 2009 & 8.1 & 68.9 & 145.2 & 26.4 & 80.9 & 24.9 & 354.4 \\
\hline 2010 & 10.7 & 93.2 & 62.6 & 77.0 & 106.3 & 109.9 & 459.7 \\
\hline $\begin{array}{c}\text { The average over the } \\
\text { years } 1987-2000\end{array}$ & 38.6 & 44.1 & 52.4 & 49.8 & 43.0 & 47.3 & 275.2 \\
\hline \multicolumn{8}{|c|}{ Temperature $\left({ }^{\circ} \mathrm{C}\right)$} \\
\hline 2008 & 9.1 & 12.7 & 17.4 & 18.4 & 18.5 & 12.2 & 14.7 \\
\hline 2009 & 10.3 & 12.9 & 15.7 & 19.4 & 17.7 & 14.6 & 15.1 \\
\hline 2010 & 8.9 & 14.0 & 17.4 & 21.6 & 19.8 & 11.8 & 15.6 \\
\hline $\begin{array}{c}\text { The average over the } \\
\text { years } 1987-2000\end{array}$ & 7.8 & 12.5 & 17.2 & 19.2 & 18.5 & 13.1 & 14.7 \\
\hline \multicolumn{8}{|c|}{ Sielianinov's hydrothermic coefficients } \\
\hline 2008 & 1.04 & 2.18 & 0.94 & 1.25 & 1.36 & 1.73 & 1.39 \\
\hline 2009 & 0.26 & 1.72 & 3.08 & 0.44 & 1.48 & 0.57 & 1.28 \\
\hline 2010 & 0.40 & 2.14 & 1.20 & 1.15 & 1.74 & 3.10 & 1.61 \\
\hline
\end{tabular}

Coefficient value [Bac at al. 1998]: <0.5 - strong drought; 0.51-0.69- semi drought; 0.70-0.99- pure drought; $\geq 1$ - fault drought.

harvest were significantly dependent on the weed control methods (Tables 2, 3).

The most effective in reducing weed infestation at both dates appeared to be variants: 3 - in which earthing-up with harrowing were used before emergence and spraying with a mixture of herbicides Command 480 EC $0.21 \cdot \mathrm{ha}^{-1}+$ Dispersive Afalon $450 \mathrm{SC} 1.01 \cdot \mathrm{ha}^{-1}$ about 7 days before emergence; and variant 5 . which means earthing-up with harrowing until emergences and spraying with mixture of herbicides Stomp 400 SC $3.51 \cdot$ ha $^{-1}+$ Dispersive Afalon $450 \mathrm{SC} 1.01 \cdot$ ha $^{-1}$ (Tables 2, 4).

The results of the study are in compliance with reports of Eberlain et al. [1997], Kraska et al. [2006], Tomczak et al. [2007], Gugała and Zarzecka [2011] as well as Ciesielska and Wysmułek [2012]. These authors gained the highest percentage of destroying weeds by using combination of at least two herbicides or their mixtures, at two dates. The result of weed control with the mixtures of herbicides was also less dependent on the meteorological conditions in the years of research than after applying one preparation.

The weather conditions in particular years of conducting the experiment had a significant impact on weed infestation of the plantation (Table 1). The highest number of weeds, before row closure as well as before tuber harvest, was found in 2008 which was characterized by even distribution of rainfall and temperatures (Tables
$4,5,6)$. However, the lowest number of weed species was found in 2009 when severe rainfall deficiency occurred in April and July. A similar impact of the weather conditions on the weed infestation of potato was observed by Gruczek [2001], Zarzecka and Gugała [2004a, b] and Sawicka et al. [2011].

The analysis of the percentage share of weed species showed that at the beginning of the potato growing season the dominant taxons were as follows: Agropyron repens (L.) - average $19.8 \%$, Echinochloa crus-galli (L.) - average $13.2 \%$, Viola arvensis (Murr.) - average $12.1 \%$, Chenopodium album (L.) - average $11.2 \%$ (Table 4). Also at the second date, before the tuber harvest, similar correlation appeared and the highest percentage share of these weed species have been seen: Agropyron repens (L.) - average $25.9 \%$ Chenopodium album (L.) - average 20.3\%, Echinochloa crus-galli (L.) - average $14.7 \%$, Polygonum convolvulus (L.) - average $11.2 \%$ (Table 5).

These taxons posed the greatest threat to growth, development and yielding of the potato. According to Kapeluszny [1980], few species characterized by eminent aggressiveness or abundant may decide about weed infestation of the field. While the abundance of species composition does not always prejudge the intensity of infestation. 
Table 2. The species composition and the number of weeds per $1 \mathrm{~m}^{2}$ before potato row closure depending on weed control methods

\begin{tabular}{|c|c|c|c|c|c|c|}
\hline \multirow{2}{*}{ Species } & \multicolumn{5}{|c|}{ Weed control methods } & \multirow{2}{*}{ Mean } \\
\hline & $1^{*}$ & $2^{*}$ & $3^{*}$ & $4^{*}$ & $5^{*}$ & \\
\hline Chenopodium album (L.) & 2.1 & 1.8 & 0.9 & 1.8 & 1.1 & 1.5 \\
\hline Thlaspi arvense (L.) & 1.6 & 3.1 & 1.0 & 1.3 & 0.7 & 1.5 \\
\hline Erodium cicutarium (L.) & 1.9 & 2.6 & 1.0 & 1.2 & 0.4 & 1.4 \\
\hline Anthemis arvensis (L.) & 0.8 & 0.1 & 0.3 & - & 0.6 & 0.4 \\
\hline Viola arvensis (Murr.) & 2.0 & 1.4 & 1.2 & 1.8 & 1.5 & 1,6 \\
\hline Polygonum convolvulus (L.) & 2.1 & 1.3 & 0.9 & 1.7 & 1.7 & 1.5 \\
\hline Cirsium arvense (L.) & 0.4 & 0.9 & 0.6 & 1.4 & - & 0.7 \\
\hline Galinsoga parviflora (Cav.) & 1.0 & 0.1 & 0.2 & 0.7 & 0.7 & 0.5 \\
\hline Galium aparine (L.) & 0.8 & - & - & 0.3 & 0.2 & 0.3 \\
\hline Total of dicotyledonous & 12.7 & 11.3 & 6.1 & 10.2 & 6.9 & 9.4 \\
\hline Agropyron repens (L.) & 4.2 & 4.2 & 1.6 & 3.0 & 3.7 & 3.3 \\
\hline Echinochloa crus-galli (L.) & 6.1 & 0.9 & 1.3 & 2.0 & 1.9 & 2.4 \\
\hline Poa annua (L.) & 0.2 & 0.4 & 1.4 & 0.3 & - & 0.5 \\
\hline Total of monocotyledonous & 10.5 & 5.5 & 4.3 & 5.3 & 5.6 & 6.2 \\
\hline Other species & 2.8 & 1.3 & 2.5 & 3.4 & 2.6 & 2.6 \\
\hline Total number of weeds & 26.0 & 18.1 & 12.9 & 18.9 & 15.1 & 18.2 \\
\hline \multicolumn{6}{|c|}{$\mathrm{NIR}_{0,05} \mathrm{LSD}_{0,05}$ - between weed control methods } & 2.4 \\
\hline
\end{tabular}

$1^{*}$ - Control object, 2* - Command 480 EC $0.2 \mathrm{dm}^{3} \cdot \mathrm{ha}^{-1}, 3^{*}$ - Command 480 EC $0.2 \mathrm{dm}^{3} \cdot \mathrm{ha}^{-1}+$ Dispersive Afalon $450 \mathrm{SC} 1.0 \mathrm{dm}^{3} \cdot \mathrm{ha}^{-1}, 4^{*}$ - Stomp $400 \mathrm{SC} 3.5 \mathrm{dm}^{3} \cdot \mathrm{ha}^{-1}, 5^{*}-$ Stomp $400 \mathrm{SC} 3.5 \mathrm{dm}^{3} \mathrm{ha}^{-1}+$ Dispersive Afalon $450 \mathrm{SC} 1.0 \mathrm{dm}^{3} \cdot \mathrm{ha}^{-1}$

Other species: Veronica triphyllos (L.), Scleranthus annuus (L.), Taraxacum campylodes (G. E. Haglund), Melandrium album (Mill.), Symphytum officinale (L.)

Table 3. The species composition and the number of weeds per $1 \mathrm{~m}^{2}$ before potato tubers harvest depending on weed control methods

\begin{tabular}{|c|c|c|c|c|c|c|}
\hline \multirow{2}{*}{ Species } & \multicolumn{5}{|c|}{ Weed control methods } & \multirow{2}{*}{ Mean } \\
\hline & $1^{*}$ & $2^{*}$ & $3^{*}$ & $4^{*}$ & $5^{*}$ & \\
\hline Chenopodium album (L.) & 4.3 & 1.5 & 1.8 & 2.6 & 1.2 & 2.3 \\
\hline Thlaspi arvense (L.) & - & - & - & - & - & - \\
\hline Erodium cicutarium (L.) & 1.1 & 1.1 & 2.3 & 1.6 & 1.2 & 1.5 \\
\hline Anthemis arvensis (L.) & - & - & - & - & - & - \\
\hline Viola arvensis (Murr.) & 1.3 & 1.1 & 1.0 & 1.2 & 0.5 & 1.0 \\
\hline Polygonum convolvulus (L.) & 3.0 & 0.9 & 0.8 & 1.3 & 1.2 & 1.4 \\
\hline Cirsium arvense (L.) & 1.4 & 2.9 & 1.0 & 0.4 & 0.7 & 1.3 \\
\hline Galinsoga parviflora (Cav.) & - & - & - & 0.2 & 0.3 & 0.1 \\
\hline Galium aparine (L.) & 0.2 & - & - & - & 0.3 & 0.1 \\
\hline Total of dicotyledonous & 11.3 & 7.5 & 6.9 & 7.3 & 5.4 & 7.7 \\
\hline Agropyron repens (L.) & 4.2 & 3.7 & 1.2 & 5.3 & 3.4 & 3.6 \\
\hline Echinochloa crus-galli (L.) & 4.3 & 2.0 & 0.5 & 1.8 & 2.2 & 2.2 \\
\hline Poa annua (L.) & 0.1 & - & 0.1 & 0.4 & 0.1 & 0.1 \\
\hline Total of monocotyledonous & 8.6 & 5.7 & 1.8 & 7.5 & 5.7 & 5.9 \\
\hline Other species & 1.1 & 0.6 & 0.1 & 1.1 & 0.9 & 0.7 \\
\hline Total number of weeds & 21.2 & 13.8 & 8.8 & 15.9 & 12.0 & 14.3 \\
\hline \multicolumn{6}{|c|}{$\mathrm{NIR}_{0,05} \mathrm{LSD}_{0,05}$ between weed control methods } & 2.7 \\
\hline
\end{tabular}

1* - Control object, 2* - Command 480 EC $0.2 \mathrm{dm}^{3} \cdot \mathrm{ha}^{-1}, 3^{*}$ - Command 480 EC $0.2 \mathrm{dm}^{3} \cdot \mathrm{ha}^{-1}+$ Dispersive Afalon $450 \mathrm{SC} 1.0 \mathrm{dm}^{3} \cdot \mathrm{ha}^{-1}, 4^{*}$ - Stomp $400 \mathrm{SC} 3.5 \mathrm{dm}^{3} \cdot \mathrm{ha}^{-1}, 5^{*}-$ Stomp $400 \mathrm{SC} 3.5 \mathrm{dm}^{3} \cdot \mathrm{ha}^{-1}+$ Dispersive Afalon $450 \mathrm{SC} 1.0 \mathrm{dm}^{3} \cdot \mathrm{ha}^{-1}$

Other species: Taraxacum campylodes (G.E.Haglund), Melandrium album (Mill.), Plantago lanceolata (L.) 
Table 4. The species composition and the number of weeds per $1 \mathrm{~m}^{2}$ before potato row closure in the years 2008-2010

\begin{tabular}{|c|c|c|c|c|c|}
\hline \multirow{2}{*}{ Species } & \multicolumn{3}{|c|}{ Years } & \multirow{2}{*}{ Mean } & \multirow{2}{*}{$\begin{array}{l}\text { Percentage } \\
\text { of species }\end{array}$} \\
\hline & 2008 & 2009 & 2010 & & \\
\hline Chenopodium album (L.) & 3.0 & 1.9 & 1.2 & 2.0 & 11.2 \\
\hline Thlaspi arvense (L.) & 2.9 & 0.2 & 0.5 & 1.2 & 6.6 \\
\hline Erodium cicutarium (L.) & 1.6 & - & 2.3 & 1.3 & 7.2 \\
\hline Anthemis arvensis (L.) & 0.2 & - & 0.4 & 0.2 & 1.1 \\
\hline Viola arvensis (Murr.) & 3.7 & 2.4 & 0.4 & 2.2 & 12.1 \\
\hline Polygonum convolvulus (L.) & 3.7 & 1.1 & 0.3 & 1.7 & 9.3 \\
\hline Amaranthus retroflexus (L.) & 0.9 & - & - & 0.3 & 1.6 \\
\hline Galinsoga parviflora (Cav.) & 0.3 & 0.1 & 0.8 & 0.4 & 2.2 \\
\hline Capsella bursa pastoris (L.) & 0.3 & - & - & 0.1 & 0.5 \\
\hline Polygonum persicaria (L) & - & 0.3 & - & 0.1 & 0.5 \\
\hline Cirsium arvense (L.) & - & - & 1.2 & 0.4 & 2.2 \\
\hline Galium aparine (L.) & - & - & 0.3 & 0.1 & 0.5 \\
\hline Other species & 0.8 & 3.0 & 2.2 & 2.0 & 10.9 \\
\hline Total of dicotyledonous & 17.4 & 9.0 & 9.6 & 12.0 & 65.9 \\
\hline Agropyron repens (L.) & 3.8 & 2.8 & 4.1 & 3.6 & 19.8 \\
\hline Echinochloa crus-galli (L.) & 5.2 & 1.1 & 1.0 & 2.4 & 13.2 \\
\hline Poa annua (L.) & - & 0.3 & 0.3 & 0.2 & 1.1 \\
\hline Total of monocotyledonous & 9.0 & 4.2 & 5.4 & 6.2 & 34.1 \\
\hline Total number of weeds & 26.4 & 13.2 & 15.0 & 18.2 & - \\
\hline Total number of species & 12.0 & 10.0 & 13.0 & 16.0 & - \\
\hline \multicolumn{5}{|l|}{$\mathrm{NIR}_{0,05} \mathrm{LSD}_{0,05}$ for years } & 1.9 \\
\hline
\end{tabular}

Other species: Veronica triphyllos (L.), Scleranthus annuus (L.), Taraxacum campylodes (G.E.Haglund), Melandrium album (Mill.), Symphytum officinale (L.)

Table 5. The species composition and the number of weeds per $1 \mathrm{~m}^{2}$ before potato tubers harvest in the years 2008-2010

\begin{tabular}{|c|c|c|c|c|c|}
\hline \multirow{2}{*}{ Species } & \multicolumn{3}{|c|}{ Years } & \multirow{2}{*}{ Mean } & \multirow{2}{*}{$\begin{array}{l}\text { Percentage } \\
\text { of species }\end{array}$} \\
\hline & 2008 & 2009 & 2010 & & \\
\hline Chenopodium album (L.) & 3.7 & 2.7 & 2.2 & 2.9 & 20.3 \\
\hline Thlaspi arvense (L.) & - & - & - & - & - \\
\hline Erodium cicutarium (L.) & 1.0 & - & 1.7 & 0.9 & 6.3 \\
\hline Anthemis arvensis (L.) & - & - & - & - & - \\
\hline Viola arvensis (Murr.) & 1.0 & 2.4 & 0.3 & 1.2 & 8.4 \\
\hline Polygonum convolvulus (L.) & 3.0 & 1.7 & 0.1 & 1.6 & 11.2 \\
\hline Amaranthus retroflexus (L.) & 0.6 & - & - & 0.2 & 1.4 \\
\hline Galinsoga parviflora (Cav.) & - & - & 0.3 & 0.1 & 0.7 \\
\hline Capsella bursa pastoris (L.) & 0.3 & - & - & 0.1 & 0.7 \\
\hline Polygonum persicaria (L.) & - & 0.3 & - & 0.1 & 0.7 \\
\hline Cirsium arvense (L.) & - & - & 1.5 & 0.5 & 3.5 \\
\hline Galium aparine (L.) & - & - & 0.3 & 0.1 & 0.7 \\
\hline Total of dicotyledonous & 9.6 & 7.1 & 6.4 & 7.7 & 53.9 \\
\hline Agropyron repens (L.) & 4.1 & 1.4 & 5.5 & 3.7 & 25.9 \\
\hline Echinochloa crus-galli (L.) & 4.9 & 0.8 & 0.8 & 2.1 & 14.7 \\
\hline Poa annua (L.) & - & - & 0.3 & 0.1 & 0.7 \\
\hline Total of monocotyledonous & 8.9 & 2.2 & 6.6 & 5.9 & 41.2 \\
\hline Other species & 1.0 & 0.2 & 0.9 & 0.7 & 4.9 \\
\hline Total number of weeds & 19.6 & 9.5 & 13.9 & 14.3 & - \\
\hline Total number of species & 9.0 & 7.0 & 11.0 & 14.0 & - \\
\hline \multicolumn{5}{|l|}{$\mathrm{NIR}_{0,05}$ LSD $_{0,05}$ dla; for: lat-years } & 1.4 \\
\hline
\end{tabular}

Other species: Equisetum arvense (L.), Lamium purpureum (L.), Matricaria chamomilla (L.), Taraxacum campylodes (G. E. Haglund), Melandrium album (Mill.), Plantago lanceolata (L.) 
Table 6. The number of weeds per $1 \mathrm{~m}^{2}$ harvest depending on weed control methods and years of the study

\begin{tabular}{|c|c|c|c|c|}
\hline \multirow{2}{*}{ Cultivars } & \multicolumn{3}{|c|}{ Years } & \multirow{2}{*}{ Mean } \\
\hline & 2008 & 2009 & 2010 & \\
\hline \multicolumn{5}{|c|}{ Before row closure } \\
\hline $1^{*}$ & 34.9 & 15.7 & 27.4 & 26.0 \\
\hline $2^{*}$ & 27.2 & 12.9 & 14.0 & 18.1 \\
\hline $3^{*}$ & 19.3 & 10.7 & 8.7 & 12.9 \\
\hline $4^{*}$ & 27.4 & 15.9 & 13.4 & 18.9 \\
\hline $5^{*}$ & 23.0 & 10.8 & 11.5 & 15.1 \\
\hline Mean & 26.4 & 13.2 & 15.0 & 18.2 \\
\hline \multicolumn{5}{|c|}{$\begin{array}{l}\text { NIR }{ }_{0,05} \text { LSD }_{0,05} \text { between years } 1.9 \\
\text { between weed control methods } 1.9-\text { in interaction between weed control methods } \times \text { years } 4.1\end{array}$} \\
\hline \multicolumn{5}{|c|}{ Before tubers harvest } \\
\hline $1^{*}$ & 26.1 & 13.1 & 24.4 & 21.2 \\
\hline $2^{*}$ & 20.0 & 9.1 & 12.4 & 13.8 \\
\hline $3^{*}$ & 12.9 & 6.6 & 6.9 & 8.8 \\
\hline $4^{*}$ & 22.0 & 10.6 & 15.2 & 15.9 \\
\hline $5^{*}$ & 17.1 & 8.1 & 10.7 & 12.0 \\
\hline Mean & 19.6 & 9.5 & 13.9 & 14.3 \\
\hline
\end{tabular}

$1 *$ - Control object, $2 *$ - Command 480 EC $0.2 \mathrm{dm}^{3} \cdot \mathrm{ha}^{-1}, 3^{*}$ - Command 480 EC $0.2 \mathrm{dm}^{3} \cdot \mathrm{ha}^{-1}+$ Dispersive Afalon 450 SC $1.0 \mathrm{dm}^{3} \cdot \mathrm{ha}^{-1}, 4^{*}$ - Stomp $400 \mathrm{SC} 3.5 \mathrm{dm}^{3} \cdot \mathrm{ha}^{-1}, 5^{*}-$ Stomp $400 \mathrm{SC} 3.5 \mathrm{dm}^{3} \cdot \mathrm{ha}^{-1}+$ Dispersive Afalon $450 \mathrm{SC} 1.0 \mathrm{dm}^{3} \cdot \mathrm{ha}^{-1}$

\section{CONCLUSIONS}

The most effective in decrease of weed infestation marked before row closure and potato tuber harvest were variations: 3 - in which mechanical weed control until emergence and sprying with mixture of herbicides Command 480 EC $0.21 \cdot$ ha $^{-1}$ + Dispersive Afalon $450 \mathrm{SC} 1.0 \mathrm{l} \cdot \mathrm{ha}^{-1}$ were used as well as variant 5 - which means mechanical weed control until emergence and sprying with mixture of herbicides Stomp 400 SC $3.51 \cdot h^{-1}+$ Dispersive Afalon $450 \mathrm{SC} 1.0 \mathrm{l} \cdot \mathrm{ha}^{-1}$ just before germination. The analysis of variances did not confirm the significant impact of potato cultivars on the number and weed species composition. The weather conditions in particular years of conducting the experiment significantly diversified weed infestation of potato plants.

\section{REFERENCES}

1. Ciesielska A., Wysmułek A. 2012. Skuteczność chwastobójcza mieszaniny herbicydów Sencor 600 SC + Titus 25 WG w ziemniakach. Prog. Plant Prot./Post. Ochr. Roślin, 52 (4), 885-888.

2. Eberlain C.V., Petersom P.E., Guttyeri M.J., Strak J.C. 1997. Efficacy and economics of cultivation wed control in potato. Weed Technology, 11 (2), 257-264.

3. Fernandez-Quintanilla C., Quadranti M., Kudsk P., Barberi P. 2008. Which future for weed science? Weed Res., 48, 297-301.

4. Ginel J., Wnukowski S., Słonimska R., Dziedzic M. 1992. Effect of Sencor (metribuzin) on the inoculum activity of potato gangrene (Phoma exiqua var. foreata). Materiały 32. Sesji Nauk. Inst. Ochr. Roślin, Cz. II., 28-32.

5. Gruczek T. 2001. Efektywne sposoby walki $\mathrm{Z}$ chwastami i ich wpływ na jakość bulw ziemniaka. Biul. IHAR, 217, 221-231.

6. Gugała M., Zarzecka K. 2011. Skuteczność i selektywność herbicydów w regulacji zachwaszczenia na plantacji ziemniaka. Biul. IHAR, 262, 103-110.

7. Guttieri M.J., Eberlein C.V. 1997. Preemergence weed control in potatoes with rimsulfuron mixtures. Weed Technol., 11, 755-761.

8. Hashim S., Marwat K. B., Hassan G. 2003. Chemical weed control efficiency in potato (Solanum tuberosum L.) under agro-climatic conditions of Peshawar. Pak. J. Weed Sci. Res., 91(1), 105-110.

9. Kapeluszny J. 1980. Zachwaszczenie upraw ziemniaka na niektórych glebach środkowo-wschodniej Polski. Część II. Struktura ilościowo-jakościowa zachwaszczenia. Annales UMCS, Sec. E, 35/36, 23-37. 
10. Kraska P., Pałys E., Kuraszkiewicz R. 2006. Zachwaszczenie łanu ziemniaka w zależności od systemu uprawy, poziomu nawożenia mineralnego i intensywności ochrony. Acta Agrophys., 8(2), 423-433.

11. Nowacki W., Podolska G. 2005. Intensywność technologii a jakość ziemiopłodów. Mat. IX Konf. Nauk. Efektywne i bezpieczne technologie produkcji roślinnej, Puławy 12 czerwca 2005, 135-140.

12. Praczyk T. 2015. Atlas chwastów. IOR Poznań. www.ior.poznan.pl.

13. Praczyk T., Skrzypczak W. 2011. Stan aktualny i kierunki rozwoju herbologii. Prog. Plant Prot./ Post. Ochr. Roślin, 51 (1), 354-363.

14. Pruszyński S. 2000. Ochrona roślin w zrównoważonym rolnictwie. Rocz. Nauk. SERiA, 2(5), 12-15.

15. Pytlarz-Kozicka M. 2002. Wpływ sposobów pielęgnowania na wysokość i jakość plonów ziemniaka. Zesz. Probl. Post. Nauk Rol., 489, 147-155.

16. Rola H. 2002. Ekologiczne i produkcyjne aspekty ochrony roślin przed chwastami. Pam. Puł., 130, 635-645.

17. Sawicka B., Barbaś P., Dąbek-Gad M. 2011. Problem zachwaszczenia w warunkach stosowania bioregulatorów wzrostu i nawożenia dolistnego w uprawie ziemniaka. Nauka Przyr. Technol., 5 (2), 1-12.

18. Sobótka W. 1999. Herbicydy - wczoraj i dziś. Prog. Plant Prot./Post. Ochr. Roślin, 39 (1), 218- 223.

19. Tomczak B., Bączkowska E., Bubiniewicz P., Górniak J. 2007. Prosulfokarb - herbicyd do ochrony zbóż i ziemniaków przed chwastami jedno i dwuliściennymi. Prog. Plant Prot./Post. Ochr. Roślin, 47 (3), 280-284.

20. Trętowski J., Wójcik R. 1988. Metodyka doświadczeń rolniczych. Wyd. WSRP Siedlce, 500 ss.

21. Wesołowski M., Kaługa W. 1989. Plonowanie i zachwaszczenie ziemniaka w plonie głównym i wtórnym w zależności od sposobu zwalczania chwastów. Rocz. Nauk Roln., 112 (3-4), 141-150.

22. Zarzecka K., Gugała M. 2004a. Kształtowanie się zachwaszczenia odmian ziemniaka w zależności od sposobu pielęgnacji. Biul. IHAR, 232, 177-184.

23. Zarzecka K., Gugała M. 2004b. Produkcyjność ziemniaka w zależności od sposobu zwalczania chwastów. Cz. I. Wpływ sposobów zwalczania chwastów na plonowanie ziemniaka. Zesz. Probl. Post. Nauk Rol., 500, 407-413. 